\title{
U.S. Hydropower Resource Assessment for Mississippi
}

\author{
Prepared by: \\ Alison M. Conner \\ James E. Francfort \\ Project Manager: \\ Ben N. Rinehart
}

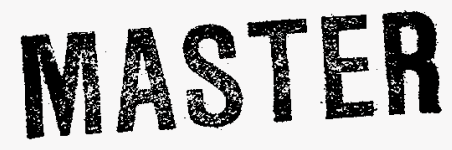

Published November 1997

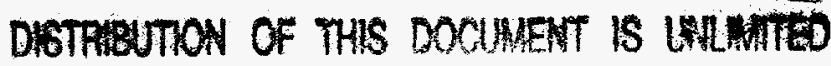

Idaho National Engineering and Environmental Laboratory

Renewable Energy Products Department

Lockheed Martin Idaho Technologies Company

Idaho Falls, Idaho $\mathbf{8 3 4 1 5}$

\author{
Prepared for the \\ U.S. Department of Energy \\ Assistant Secretary for Energy Efficiency and Renewable Energy \\ Under DOE Idaho Operations Office \\ Contract DE-AC07-94ID13223
}




\section{DISCLAIMER}

This report was prepared as an account of work sponsored by an agency of the United States Government. Neither the United States Government nor any agency thereof, nor any of their employees, makes any warranty, express or implied, or assumes any legal liability or responsibility for the accuracy, completeness, or usefulness of any information, apparatus, product, or process disclosed, or represents that its use would not infringe privately owned rights. Reference herein to any specific commercial product, process, or service by trade name, trademark, manufacturer, or otherwise does not necessarily constitute or imply its endorsement, recommendation, or favoring by the United States Government or any agency thereof. The views and opinions of authors expressed herein do not necessarily state or reflect those of the United States Government or any agency thereof. 


\section{DISCLAIMER}

Portions of this document may be illegible electronic image products. Images are produced from the best available original document. 


\begin{abstract}
The U.S. Department of Energy is developing an estimate of the undeveloped hydropower potential in the United States. The Hydropower Evaluation Software (HES) is a computer model that was developed by the Idaho National Engineering Laboratory ${ }^{a}$ for this purpose. HES measures the undeveloped hydropower resources available in the United States, using uniform criteria for measurement. The software was developed and tested using hydropower information and data provided by the Southwestern Power Administration. It is a menu-driven program that allows the personal computer user to assign environmental attributes to potential hydropower sites, calculate development suitability factors for each site based on the environmental attributes present, and generate reports based on these suitability factors. This report describes the resource assessment results for the State of Mississippi.
\end{abstract}

a. In January 1997, the name of the Idaho National Engineering Laboratory (INEL) was changed to the Idaho National Engineering and Environmental Laboratory (INEEL). INEEL will be used throughout the text of the document, except where the use of INEL is historically important. 


\section{ACKNOWLEDGMENTS}

The authors thank Peggy A. M. Brookshier and John V. Flynn of the U.S. Department of Energy, and Charles Clevenger of the State of Mississippi for their active participation and timely comments. 


\section{CONTENTS}

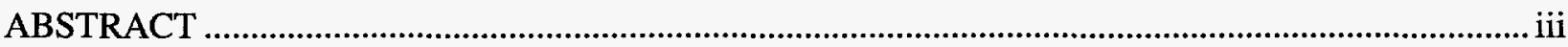

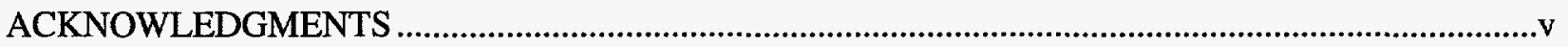

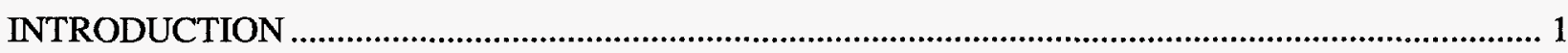

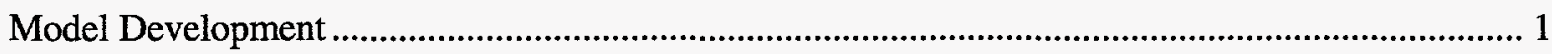

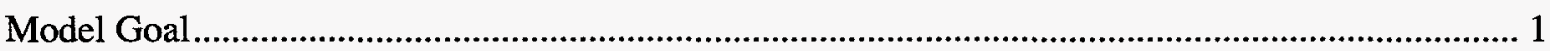

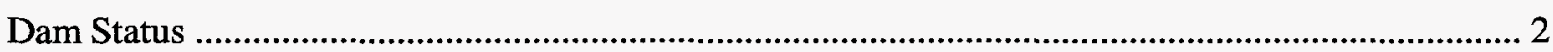

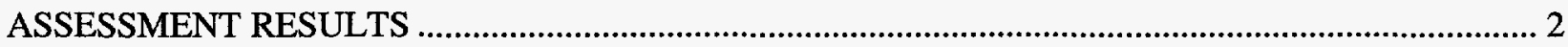

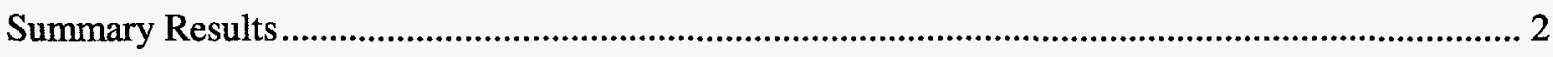

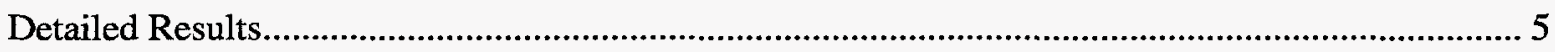

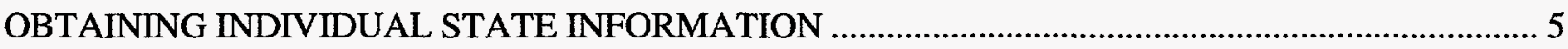

ADDITIONAL HYDROPOWER EVALUATION SOFTWARE INFORMATION .............................. 6

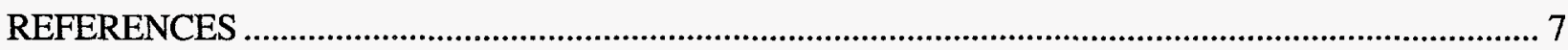

Appendix A-Summary Report

Appendix B-River Basins Report

Appendix C-Mississippi Sites List

Appendix D-Individual Resource Database List

\section{FIGURES}

1. Number of sites, by capacity groups, with HES-modeled undeveloped hydropower potential.......... 3

2. The nonmodeled and HES-modeled undeveloped hydropower potential ...................................... 3

3. The number of sites with undeveloped hydropower potential and the total megawatts of HES-modeled undeveloped hydropower potential. 
4. Number of sites with undeveloped hydropower potential in the Mississippi river basins

5. Megawatts of HES-modeled undeveloped hydropower potential in the Mississippi river basins...... 5

\section{TABLES}

1. Undeveloped hydropower potential summary for Mississippi.................................................. 2 


\section{U.S. Hydropower Resource Assessment \\ for \\ Mississippi}

\section{INTRODUCTION}

In June 1989, the U.S. Department of Energy initiated the development of a National Energy Strategy to identify the energy resources available to support the expanding demand for energy in the United States. Public hearings conducted as part of the strategy development process indicated that undeveloped hydropower resources were not well defined. As a result, the Department of Energy established an interagency Hydropower Resource Assessment Team to ascertain the undeveloped hydropower potential. In connection with these efforts by the Department of Energy, the Idaho National Engineering Laboratory designed the Hydropower Evaluation Software (HES), which has been used to perform a resource assessment of the undeveloped conventional hydropower potential in over 30 states. This report presents the results of the hydropower resource assessment for the State of Mississippi. Undeveloped pumped storage hydropower potential is not included.

The HES was developed as a tool to measure undeveloped hydropower potential regionally or by state. The software is not intended to provide precise development factors for individual sites, but to provide regional or state totals. Because the software was developed as a generic measurement tool encompassing national issues, regional and state totals must be considered judiciously; various local issues may skew undeveloped hydropower potential totals. The information for the resource assessment was compiled from the Federal Energy Regulatory Commission's Hydroelectric Power Resources Assessment database and several other sources. Refer to DOE/ID-10338, the User's Manual (Francfort, Matthews, Rinehart 1991) for the specifics of the software and to DOE/ID-10430.1, the Status Report (Conner, Francfort, Rinehart 1996) for an overview of all resource assessment activities to date.

\section{Model Development}

Hydropower Evaluation Software, both a probability-factor computer model and a database, is a menu-driven program that is intended to be user-friendly. Computer screens and report-generation capabilities were developed to meet the needs of users nationwide. The software uses environmental attribute data to generate an overall project environmental suitability factor (PESF) between 0.1 and 0.9 , where 0.9 indicates the highest likelihood of development and 0.1 indicates the lowest likelihood of development. The suitability factors depend on the unique environmental attributes of each potential site. They reflect the considerations that (a) environmental concerns can make a potential site unacceptable, prohibiting its development (for a suitability factor of 0.1 , or (b) if there are no environmental concerns, there is no negative effect on the likelihood of site development (for a suitability factor of 0.9). A combination of attributes can result in a lower suitability factor because multiple environmental considerations would reduce the likelihood that a site may be developed to its physical potential.

\section{Model Goal}

The goal of the HES is to assemble an accurate resource database of all sites with undeveloped hydropower potential in the United States for use as a planning tool to determine the viable national hydropower potential. Undeveloped hydropower potential is not limited to the development of new sites; it also includes the development of additional hydropowergenerating capacity at sites that currently have hydropower, but are not developed to their full potential. This undeveloped hydropower potential is a source of nonpolluting, renewable energy available to meet the growing power needs of the United States. The HES should help make this goal obtainable and ensure a set of uniform criteria for national assessment. 


\section{Dam Status}

The effects of environmental attributes vary by dam status. The dam status classifications used are as follows:

$$
\begin{aligned}
& W=\text { Developed hydropower site } \\
& \text { with current power generation, } \\
& \text { but the total hydropower po- } \\
& \text { tential has not been fully de- } \\
& \text { veloped. Only the undeveloped } \\
& \text { hydropower potential is dis- } \\
& \text { cussed in this report. }
\end{aligned}
$$

$\mathrm{W} / \mathrm{O}=$ Developed site without current power generation. The site has some type of developed impoundment or diversion structure, but no developed hydropower generating capability.

$\mathrm{U}=\underline{\text { Undeveloped site. The site }}$ does not have power generation capability nor a developed impoundment or diversion structure.

\section{ASSESSMENT RESULTS Summary Results}

A total of 19 sites (Table 1) have been identified and assessed for their undeveloped hydropower potential. The HES results for individual site capacities range from 30 kilowatts $(\mathrm{kW})$ to 16 megawatts (MW). The majority $(84 \%)$ of the sites in Mississippi are less than $10 \mathrm{MW}$. (Figure 1).

The nonmodeled undeveloped hydropower potential total for Mississippi was identified as $128 \mathrm{MW}$. The HES results lowers this estimate about $28 \%$ to $92 \mathrm{MW}$. The State of Mississippi does not have any hydropower sites with current power generation. The greatest reduction in undeveloped hydropower potential, by MW, occurs at developed sites without current power generation capability (W/O). These sites have a HES-modeled undeveloped hydropower potential of $62 \mathrm{MW}$, a $19-\mathrm{MW}$ reduction in the estimated undeveloped hydropower potential (Figure 2). Figure 3 correlates the number of sites that have undeveloped hydropower potential with the total megawatts of HESmodeled undeveloped hydropower potential

The 19 identified sites are located within 3 major river basins and several minor river basins. The number of sites per major river basin ranges from 2 in the Pearl River Basin to 7 in the Tombigbee River Basin (Figure 4). The major river basins within Mississippi have similar amounts of undeveloped hydropower potential. Sixty-four percent of the undeveloped hydropower potential in the State of Mississippi lies within the minor river basin sites (Figure 5).

Table 1. Undeveloped hydropower potential summary for Mississippi. The table contains the nonmodeled undeveloped nameplate potential and the HES-modeled undeveloped potential totals.

$$
\text { Number of projects Nameplate potential HES-modeled potential }
$$

\begin{tabular}{lrcc} 
& & $(\mathrm{MW})$ & $(\mathrm{MW})$ \\
\cline { 2 - 4 } With Power & 0 & 0 & 0 \\
W/O Power & 13 & 81.3 & 62.2 \\
Undeveloped & 6 & 46.7 & 29.4 \\
State Total & 19 & 128.0 & 91.6 \\
\hline
\end{tabular}




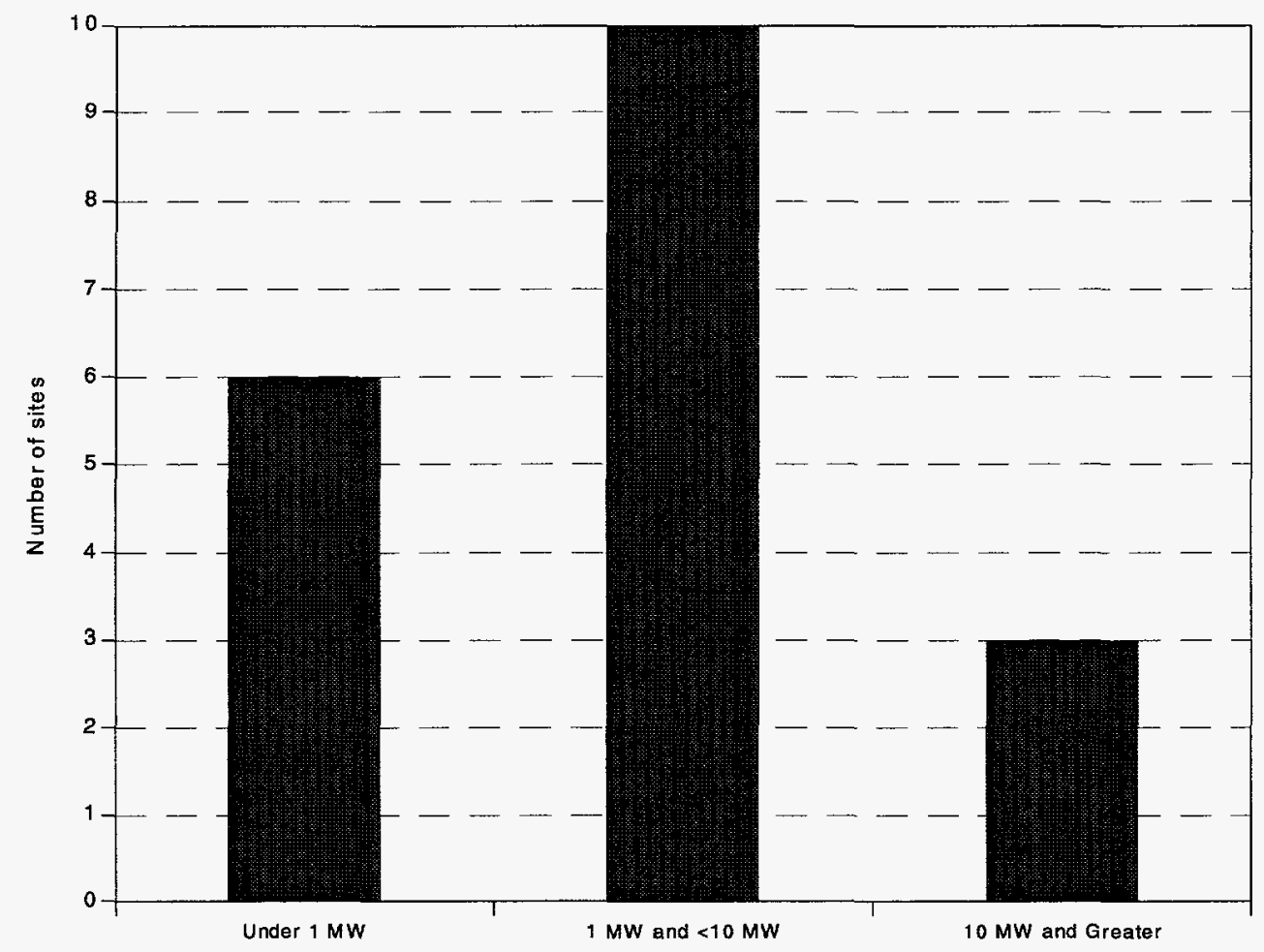

Figure 1. Number of sites, by capacity groups, with HES-modeled undeveloped hydropower potential.

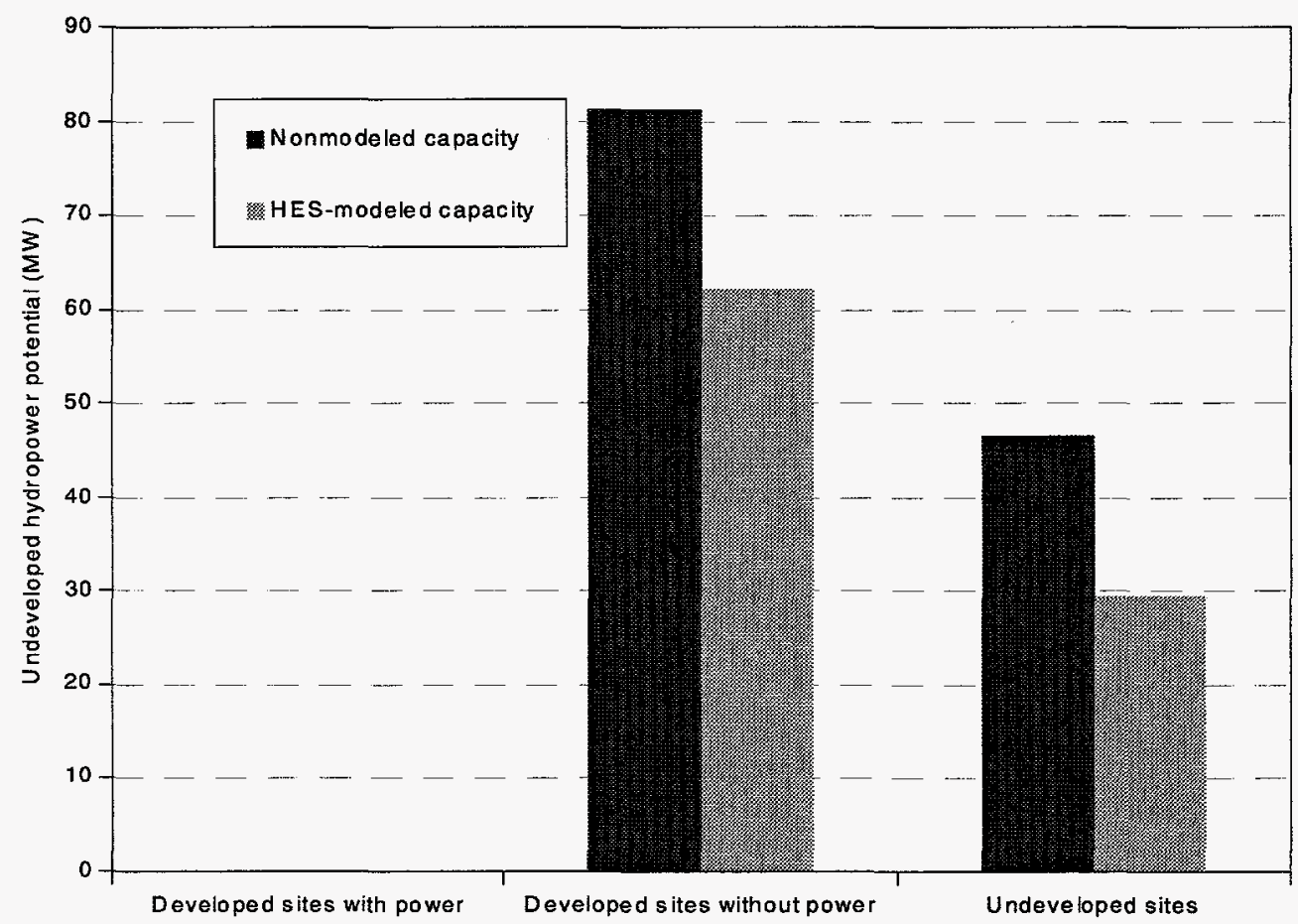

Figure 2. The nonmodeled and HES-modeled undeveloped hydropower potential. 


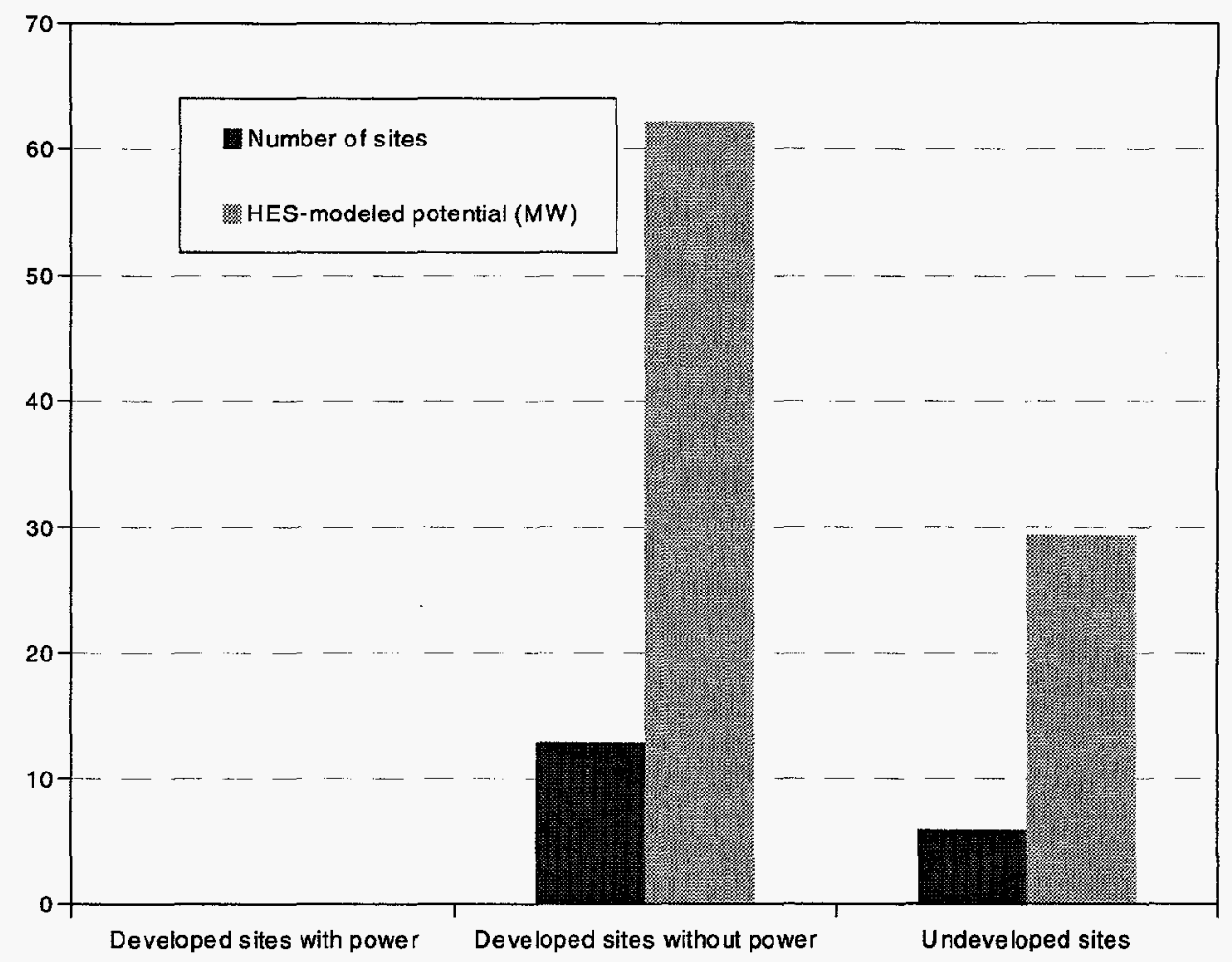

Figure 3. The number of sites with undeveloped hydropower potential and the total megawatts of HES-modeled undeveloped hydropower potential.

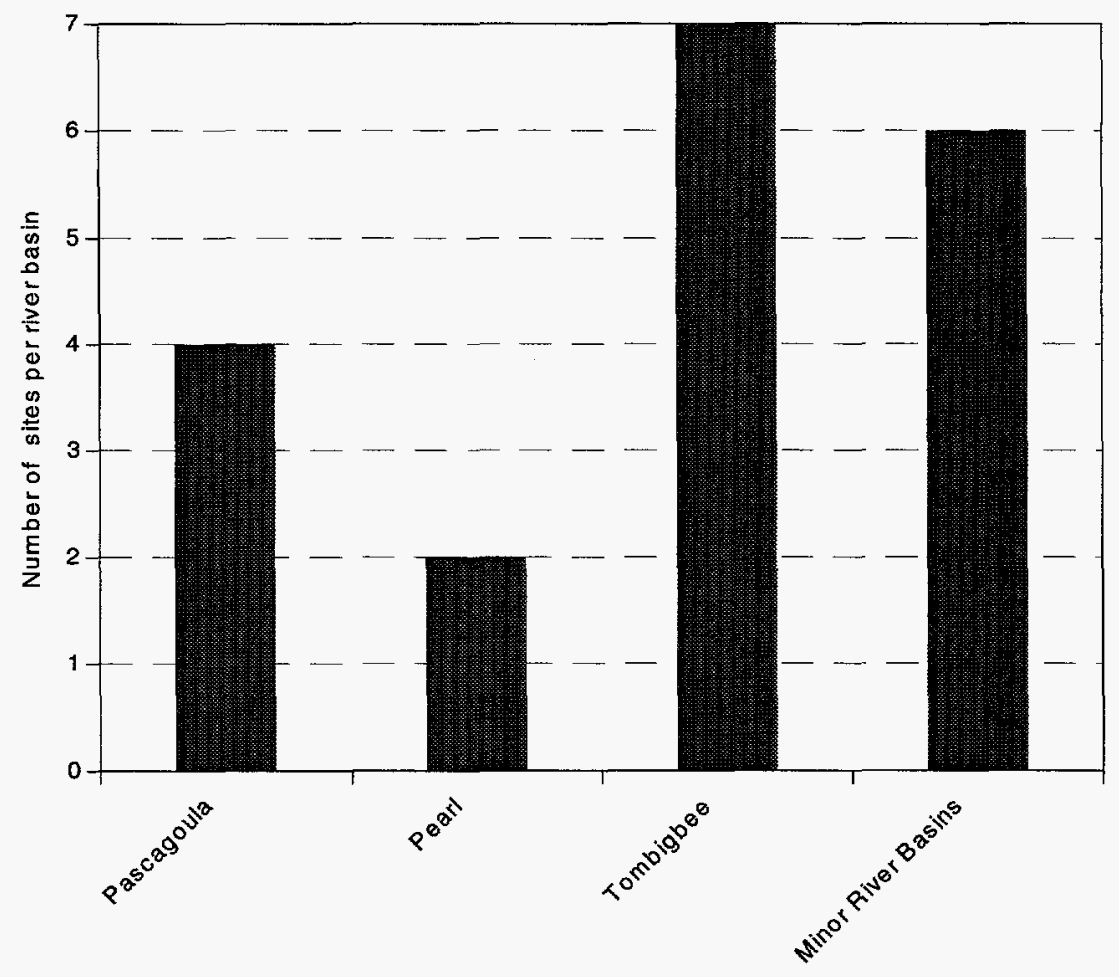

Figure 4. Number of sites with undeveloped hydropower potential in the Mississippi river basins. 


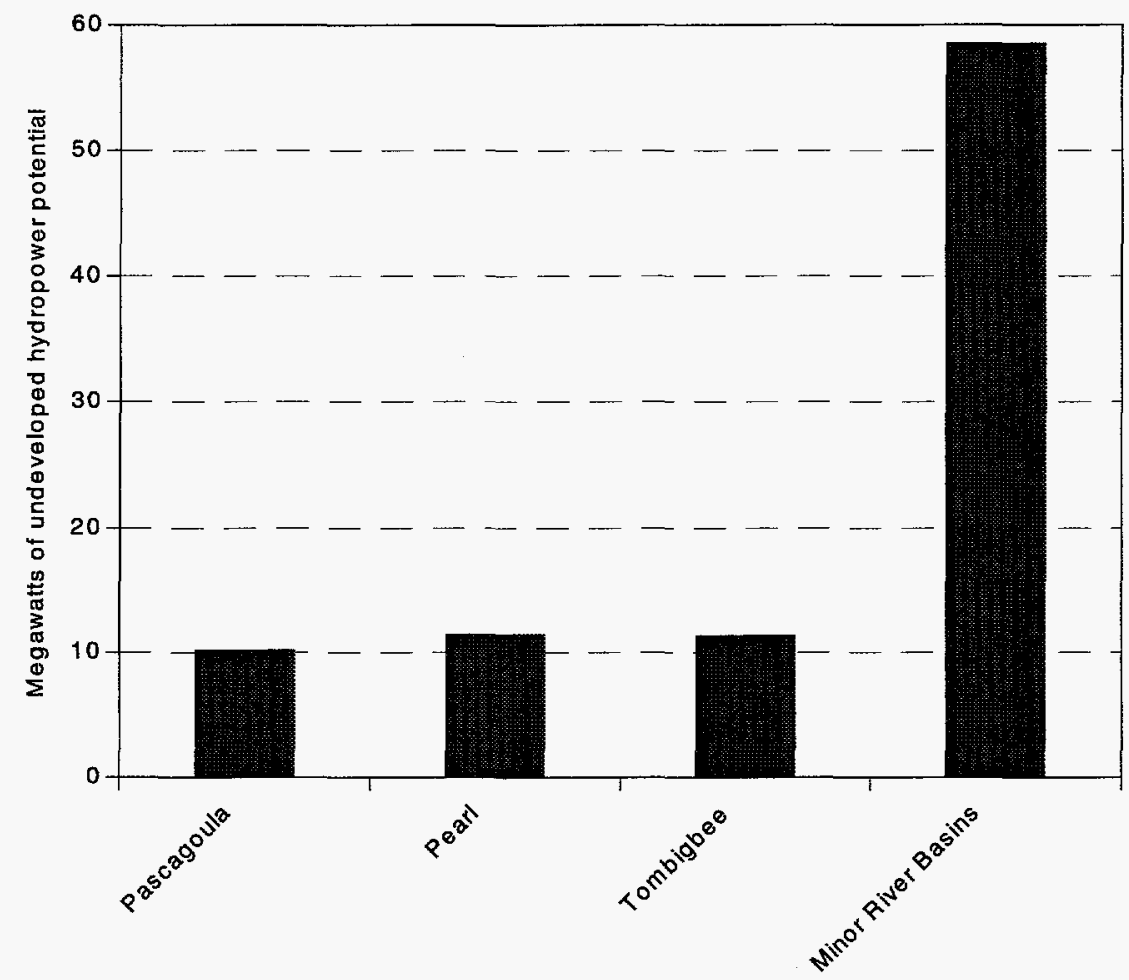

Figure 5. Megawatts of HES-modeled undeveloped hydropower potential in the Mississippi river basins.

\section{Detailed Results}

The appendices contain, in the form of HES-generated reports, detailed information about the undeveloped hydropower potential in Mississippi. The appendices contain the following information:

Appendix A summarizes the undeveloped hydropower potential by dam status groups. The number of sites, nonmodeled undeveloped hydropower potential, and HES-modeled undeveloped hydropower potential is provided based on the dam status.

Appendix B provides the hydropower resource assessment by river basin, which includes the project number, project name, stream name, dam status, nonmodeled undeveloped hydropower potential, and the HES-modeled undeveloped hydropower potential for each site. Subtotals are provided for each river basin.

Appendix C lists the project numbers, plant name, stream name, if a site is Federally owned, nonmodeled undeveloped hydropower potential, and HES-modeled undeveloped hydropower potential. The sites are grouped by dam status.

Appendix D contains a resource database list for the 19 sites in Mississippi. Information includes plant name, stream, state, county, river basin and owner names, project number, nameplate and HES-modeled undeveloped hydropower potential, the unit and plant types, dam status, latitude, longitude, and the environmental factors that the HES uses to determine the PESF.

\section{OBTAINING INDIVIDUAL STATE INFORMATION}

Additional copies of the hydropower resource assessment results for individual states are available and can be obtained by writing or calling the authors or the National Technical Information Service (NTIS).

Telephone Orders-(703) 487-4650. NTIS sales desk and customer services are available 
between 8:30 a.m. and 5:00 p.m., Eastern Standard Time.

Fax-(703) 321-8547. Customers may fax their orders to NTIS. These orders may be charged to a NTIS deposit account, American Express, VISA, or MasterCard.

Mail Orders-Mail orders should be sent to National Technical Information Service, Document Sales, 5285 Port Royal Road, Springfield, VA 22161. Call the sales desk for prices before placing an order.

E-mail-Customers may e-mail their requests toinfo@ntis.fedworld.gov.

Method of Payment-Customers may pay for reports (and other NTIS products and services) by (a) credit card (American Express, Visa or MasterCard); (b) check or money order on a United States bank payable to NTIS; (c) NTIS deposit account; or, (d) by asking to be billed (add $\$ 7.50$ per order), United States, Canada, and Mexico, only.

Handling Fee- A $\$ 4.00$ handling fee per total order applies to orders from the United States, Canada, and Mexico. Handling charges do not apply to rush order service or pick-up orders.

Postage and Shipping-Orders are shipped first class mail, or equivalent, to addresses in the United States, Canada, and Mexico.

Order Turnaround Time-Orders for technical reports generally are shipped within 3 to 5 days of receipt. For faster service, NTIS offers rush order service.

Rush Order Service-Call 1-800-533-NTIS. In Virginia, Canada, and Mexico call (703) 487-4700. For NTIS rush order service add $\$ 15$ per item. This guarantees that an order will be processed through NTIS within 24 hours of its receipt. These orders receive immediate, individual attention. The items ordered are delivered by first call mail. Call NTIS for information on rush order service for computer products.

For Help in Tracing an Order-Call (703) $487-4650$ and request the customer service option.

\section{ADDITIONAL HYDROPOWER EVALUATION SOFTWARE INFORMATION}

Additional information concerning the HES can be obtained by contacting Ben Rinehart or Jim Francfort at the addresses provided below. Copies of the software and the User's Manual may also be obtained from these individuals.

Ben Rinehart, Project Manager

Idaho National Engineering and Environmental Laboratory

P.O. Box 1625, MS 3830

Idaho Falls, ID 83415-3830

(208) 526-1002

Jim Francfort

Idaho National Engineering and Environmental Laboratory

P.O. Box 1625, MS 3830

Idaho Falls, ID $83415-3830$

(208) 526-6787

Information concerning the State of Mississippi's involvement with the resource assessment or about the identified sites may be obtained by contacting:

Charles Clevenger

State of Mississippi

Chief, Dam Safety Division

Department of Environmental Quality

P.O. Box 10631

Jackson, MS 39289-0831

(601) 961-5204 


\section{REFERENCES}

Conner, A. M., J. E. Francfort, and B. N. Rinehart, 1996, Uniform Criteria for U.S. Hydropower Resource Assessment, Hydropower Evaluation Software Status Report-II, DOE/ID 10430.1, Idaho National Engineering Laboratory, Idaho Falls, Idaho.

Francfort, J. E., S. D. Matthews, and B. N. Rinehart, 1991, Hydropower Evaluation Software User's Manual, DOE/ID-10338, Idaho National Engineering Laboratory, Idaho Falls, Idaho. 
Appendix A

Summary Report 
DATE: $10 / 17 / 97$

H Y D R O P O W E R

Number of

state

Category

With Power

W/O Power

Undeveloped

STATE

TOTAL
13

6

19
C A P A C I T Y

Name Plate

Capacity (KW)

0

81320

46700

128020
HES Adjusted capacity (KW)
S U M M A R Y
0

62247.50

29390

91637.50 


\section{Appendix B}

\section{River Basins Report}


Page No.

$10 / 17 / 97$

FERC

Number

** River Basin MINOR RIVER BASINS

07566 SUNFLOWER L\&D 1

BIG SUNFLOWER $R$

MSO05 YOUNGTON

BIG BLACK R

10410 ARKABUTLA

COLDWATER $R$

10411 ENID

YACONA R

10412 GRENADA

YALOBUSHA $R$

10413 SARDIS

IITTLE TALLAHATCHIE R

** Subtotal **

** River Basin PASCAGOULA RIVER BASIN

MSOO3 HARLESTON

ESCATAWPA R

06607 OKATIBBEE DAM

OKATIBBEE CR, CHICKASAWHAY R

MSO01 ARCHUSA CREEK

ARCHUSA CR, CHICKASAWHAY R

MSO02 FLINT CREEK DAM

FLINT CR, RED CR

** Subtotal **

** River Basin PEARI RIVER BASIN

MSOO4 YOCKANOOKANY

YOCKANOOKANY $R$, PEARI $R$

07895 ROSS BARNETT

PEARI R

** Subtotal **
$\mathrm{U}$

Dam Name Plate

stat Rating (KW) PESF

PESF * KW

U

wo

wo

Wo

wo

$21000.00 \quad 0.75$

87200.00

58520.00

U

wo

wo

wo

$11000.00 \quad 0.90$

$500.00 \quad 0.50$

$110.00 \quad 0.50$

$60.00 \quad 0.50$

30.00

11670.00

10235.00

$\mathrm{U}$

$3300.00 \quad 0.90$

2970.00

wo

$9500.00 \quad 0.90$

8550.00

12800.00

11520.00 
Page No.

$10 / 17 / 97$

FERC

Number

HYDROPOWER RESOURCE ASSESSMENT BY RIVER BASIN

Plant Name/

Stream
Dam Name Plate
Stat Rating (KW) PESF

PESF * KW

** River Basin TOMBIGBEE RIVER BASIN

09209 TENN-TOM LOCK D

TOMBIGBEE $R$

09210 TENN-TOM LOCK E

TOMBIGBEE $\mathrm{R}$

07232 COLUMBUS L\&D

TOMBIGBEE $R$

07233 ABERDEEN L\&D

TOMBIGBEE $R$

09211 TENN-TOM LOCK C

TOMBIGBEE $R$

09212 TENN-TOM LOCK B(2)

TOMBIGBEE $R$

09213 TENN-TOM LOCK A TOMBIGBEE $R$
U

U

wo

wo

wo

พั

wo
$1800.00 \quad 0.50$

$1800.00 \quad 0.50$

$3600.00 \quad 0.75$

$3600.00 \quad 0.75$

$1500.00 \quad 0.75$

$2250.00 \quad 0.75$

1800.00

0.75

16350.00

128020.00
900.00

900.00

2700.00

2700.00

1125.00

1687.50

1350.00

11362.50

91637.50 
Appendix C

Mississippi Sites List 
Page No.

$10 / 17 / 97$
1

HYDROPOWER RESOURCE ASSESSMENT BY FERC NUMBER

Plant Name/

stream

Dam Name Plate

ST Stat Rating (KW) PESF

PESF * KW

** FERC Number 07566

SUNFLOWER L\&D 1

BIG SUNFLOWER $R$

MS U

$800.00 \quad 0.90$

720.00

** Subtotal **

** FERC Number 09209

TENN-TOM LOCK D

TOMBIGBEE $R$

** Subtotal **

** FERC Number 09210

TENN-TOM LOCK E

TOMBIGBEE $R$

** Subtotal **

** FERC Number MS003

HARLESTON

ESCATAWPA R

MS U

$$
800.00
$$

720.00

MS U

$1800.00 \quad 0.50$

900.00

1800.00

900.00

MS U

$1800.00 \quad 0.50$

900.00

1800.00

900.00

** Subtotal **

** FERC Number MSO04

YOCKANOOKANY

YOCKANOOKANY R, PEARL $R$

MS U

** Subtotal **

** FERC Number MSO05

YOUNGTON

BIG BLACK R

MS U

** Subtotal **

** FERC Number 06607

OKATIBBEE DAM

OKATIBBEE CR, CHICKASAWHAY R
MS wo
11000.00

9900.00

$3300.00 \quad 0.90$

2970.00

3300.00

2970.00

9900.00

$11000.00 \quad 0.90$

(2970.00

14000.00

$28000.00 \quad 0.50$

14000.00

28000.00

$500.00 \quad 0.50$

250.00 
Page No.

HYDROPOWER RESOURCE ASSESSMENT BY FERC NUMBER Plant Name/
stream

** Subtotal **

** FERC Number 07232

COLUMBUS L\&D

TOMBIGBEE $\mathrm{R}$

** Subtotal **

** FERC Number 07233

ABERDEEN L\&D

TOMBIGBEE $\mathrm{R}$

** Subtotal **

** FERC Number 07895

ROSS BARNETT

PEARL R

** Subtotal **

** FERC Number 09211

TENN-TOM LOCK C

TOMBIGBEE $R$

** Subtotal **

** FERC Number 09212

TENN-TOM LOCK B(2)

TOMBIGBEE $\mathrm{R}$

** Subtotal **

** FERC Number 09213

TENN-TOM LOCK A

TOMBIGBEE $\mathrm{R}$

** Subtotal ** $\begin{array}{ll}\text { Dam } & \text { Name Plate } \\ \text { ST Stat Rating (KW) PESF } & \text { PESF * KW }\end{array}$

$$
500.00
$$

250.00

MS Wo

3600.00

0.75

2700.00

3600.00

2700.00

MS Wo

$3600.00 \quad 0.75$

2700.00

3600.00

2700.00

MS WO

$9500.00 \quad 0.90$

8550.00

9500.00

8550.00

MS wo

1500.00

0.75

1125.00

1500.00

1125.00

MS Wo

$2250.00 \quad 0.75$

1687.50

2250.00

1687.50

MS Wo

1800.00

0.75

1350.00

1800.00

1350.00 
HYDROPOWER RESOURCE ASSESSMENT BY FERC NUMBER

Plant Name/

Stream

** FERC Number 10410

ARKABUTLA

COLDWATER $R$

** Subtotal **

** FERC Number 10411

ENID

YACONA $R$

** Subtotal **

** FERC Number 10412

GRENADA

YALOBUSHA $R$

** Subtotal **

** FERC Number 10413

SARDIS

LITTLE TALLAHATCHIE R

** Subtotal **

** FERC Number MSOO1

ARCHUSA CREEK

ARCHUSA CR, CHICKASAWHAY $R$

** Subtotal **

** FERC Number MSO02

FLINT CREEK DAM

FLINT CR, RED CR

** Subtotal **

*** Total *** $\begin{array}{lll}\text { Dam } & \text { Name Plate } & \\ \text { ST Stat } & \text { Rating (KW) PESF } & \text { PESF * KW }\end{array}$

MS wo

$10000.00 \quad 0.75$

7500.00

10000.00

7500.00

MS wo

10400.00

0.75

7800.00

10400.00

7800.00

MS พั

$17000.00 \quad 0.75$

12750.00

17000.00

12750.00

MS wo

$21000.00 \quad 0.75$

15750.00

21000.00

15750.00

MS Wo

$$
110.00 \quad 0.50
$$

55.00

110.00

55.00

MS wo

$$
60.00 \quad 0.50
$$

30.00

60.00

30.00

128020.00

91637.50 


\section{Appendix D}

Individual Resource Database List 
R E S O U R C E

DATE: $10 / 17 / 97$

FERC

Number

Plant Name

06607 OKATIBBEE DAM

county Name

LAUDERDALE

Class

Owner Name

$R$ CORPS OF ENGINEERS-MOBILE DISTRICT

Name Plate

Rating (KW)

PESF

500.00

0.50

250.00

Plant

Type

Project status

C

ROR

$\mathrm{XX}$

Exist Prob

Factor

Wild/Scenic Protection

wild/Scenic Tributary or

Upstream / Downstream

wild/Scenic Location

Cultural Value

Fish Presence Value

Geologic Value

Historic Value

other Value

Recreation Value

Scenic Value
D A T A B A S E

I I $S I N G$

PAGE NO: 1

State

Name

OKATIBBEE CR, CHICKASAWHAY $R$

MS

River Basin

PASCAGOULA RIVER BASIN
Annual Energy
PESF Annual Energy Rating (MWh)
1050

$$
2100.00
$$

$\begin{array}{lll}\text { Dam } & \text { Latitude } & 3227.00 \\ \text { Status } & \text { Longitude } & 8850.00 \\ \text { wo } & \end{array}$

Exist Prob

$$
0.90
$$

Wildlife Value

Threatened/Endangered Fish Threatened/Endangered Wildlife

0.90 Federal Land Code 103

0.90 Federal Land Code 104

0.90 Federal Land Code 105

0.90 Federal Land Code 106

0.90 Federal Land Code 107

0.90 Federal Land Code 108

0.90 Federal Land Code 198
0.90

0.90

0.90

0.90

$\mathrm{Y} \quad 0.75$

0.90

0.90

0.90

0.90

0.90 
DATE: $10 / 17 / 97$

PAGE NO: 2

FERC

Number

Plant Name

Stream

State

07232 COLUMBUS L\&D

TOMBIGBEE R

Name

MS

county Name

LOWNDES
River Basin

TOMBIGBEE RIVER BASIN

Class Owner Name

R CORPS OF ENGINEERS-MOBILE DISTRICT

Name Plate

Rating (KW)

3600.00
PESF

$0.75 \quad 2700.00$

Unit

Type

C

$$
\begin{aligned}
& \text { Plant } \\
& \text { Type } \\
& \text { ROR }
\end{aligned}
$$

Factor

Exist Prob

0.90

Wild/Scenic Protection

Wild/Scenic Tributary or Upstream/Downstream wild/Scenic Location

Cultural value

Fish Presence Value

Geologic Value

Historic Value

Other Value

Recreation Value

Scenic Value
Project

status

$\mathrm{XX}$

Dam

Status

wo

\section{Annual Energy} Rating (MWh)

17450.00

Latitude

Longitude
PESF Annual Energy Rating (MWh)

13087.50 
DATE: $10 / 17 / 97$

FERC

Number

07233 ABERDEEN L\&D

county Name

MONROE

Class

R CORPS OF ENGINEERS-MOBILE DISTRICT

Name Plate

Rating (KW)

PESF

PESF*KW

Annual Energy

3600.00

0.75

2700.00

Project status

$\mathrm{xx}$

Exist Prob

Factor

Plant

ROR

Unit
Type

Type

C

\section{Factor}

wild/Scenic Protection

Wild/Scenic Tributary or

Upstream/Downstream

wild/Scenic Location

Cultural Value

Fish Presence Value

Geologic Value

Historic Value

other Value

Recreation value

Scenic Value

\author{
River Basin \\ TOMBIGBEE RIVER BASIN
}

Owner Name

$$
18250.00
$$

$\begin{array}{lll}\text { Dam } & \text { Latitude } & 3350.00 \\ \text { Status } & \text { Longitude } & 8831.00\end{array}$

wo
0.90 Wildlife Value Threatened/Endangered Fish Threatened/Endangered Wildlife

0.90 Federal Land Code 103

0.90 Federal Land Code 104

0.90 Federal Land code 105

0.90 Federal Land Code 106

0.90 Federal Land Code 107

0.90 Federal Land Code 108

0.90 Federal Land Code 198
PAGE NO: 3

State

Name

TOMBIGBEE $\mathrm{R}$

MS

PESF Annual Energy Rating (MWh)

13687.50

Exist Prob

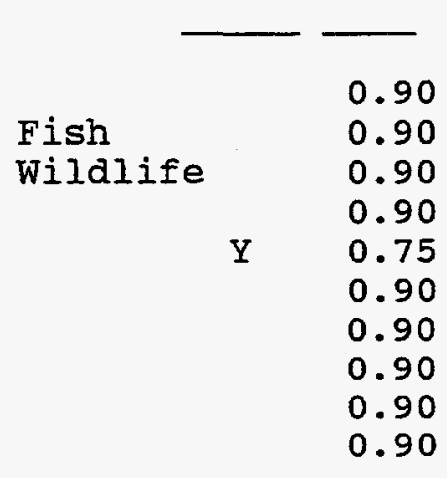


DATE: $10 / 17 / 97$

FERC

Number

Plant Name

07566 SUNFLOWER L\&D 1

County Name

WASHINGTON

Class

R

D\&W JONES, INC

Name Plate

Rating ( $\mathrm{KW}$ )

PESF

Annual Energy

800.00

$0.90 \quad 720.00$

PESF*KW

Rating (MWh)

2880.00

Dam

Unit

Plant

Type

Type

C

STG

Factor

Exist Prob

0.90

Wildlife Value

Threatened/Endangered Fish

Wild/Scenic Tributary or Upstream/ Downstream wild/Scenic Location

Cultural Value

Fish Presence Value

Geologic Value

Historic Value

other value

Recreation value

Scenic Value

$\mathrm{U}$

Factor

Threatened/Endangered Wildlife

0.90 Federal Land Code 103

0.90 Federal Land Code 104

0.90 Federal Land Code 105

0.90 Federal Land Code 106

0.90 Federal Land Code 107

0.90 Federal Land Code 108

0.90 Federal Land Code 198

0.90
PAGE NO: 4

State

Name

MS
PESF Annual

Energy Rating (MWh)

2592

Exist Prob

0.90

0.90

0.90

0.90

0.90

0.90

0.90

0.90

0.90

0.90 
DATE: $10 / 17 / 97$

FERC

Number

07895

ROSS BARNETT

County Name

RANKIN

Class

Owner Name

R PEARL RIVER VALLEY WATER DISTRICT
River Basin

PEARL RIVER BASIN
PAGE NO: 5

State

Name

PEARL $R$

MS

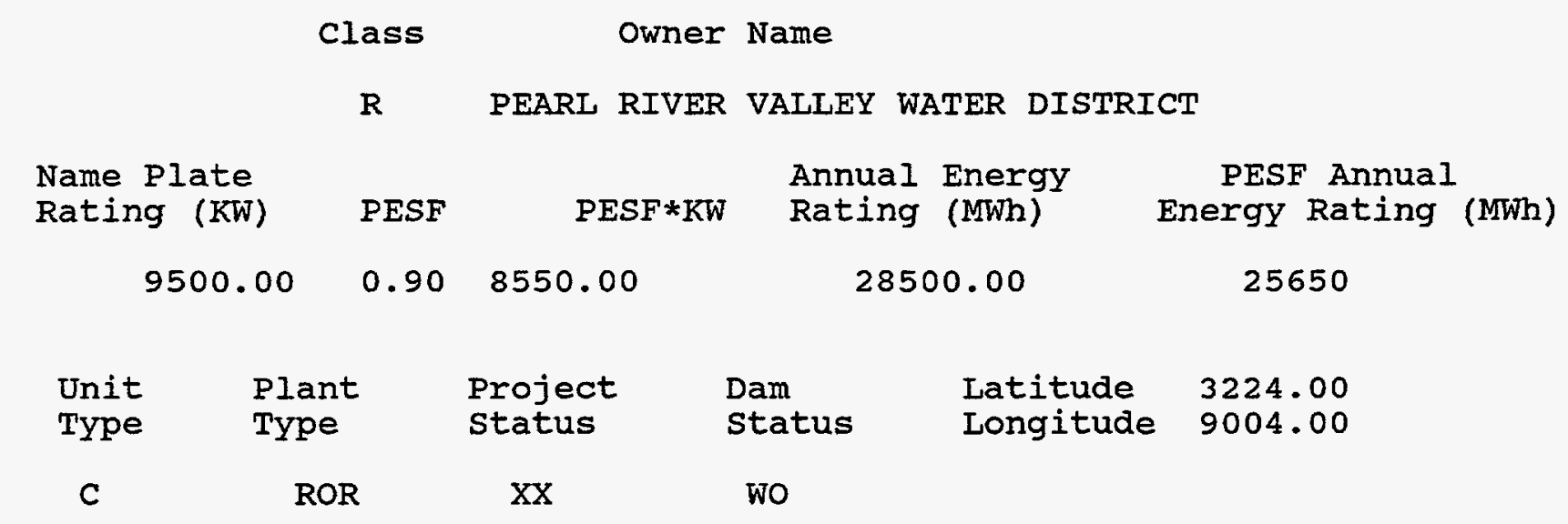

Factor

Wild/Scenic Protection

wild/Scenic Tributary or Upstream/Downstream wild/Scenic Location Cultural Value Fish Presence Value Geologic Value Historic Value other Value Recreation value Scenic Value
Exist Prob

0.90

Wildlife Value Threatened/Endangered Fish Threatened/Endangered Wildlife

0.90 Federal Land Code 103

0.90 Federal Land Code 104

0.90 Federal Land Code 105

0.90 Federal Land Code 106

0.90 Federal Land Code 107

0.90 Federal Land Code 108

0.90 Federal Land Code 198
Exist Prob

0.90

0.90

0.90

0.90

0.90

0.90

0.90

0.90

0.90

0.90 
DATE: $10 / 17 / 97$

PAGE NO: 6

FERC

Number

Plant Name

stream

State

09209 TENN-TOM LOCK D

TOMBIGBEE $R$

Name

09209

county Name

River Basin

ITAWAMBA

TOMBIGBEE RIVER BASIN

MS

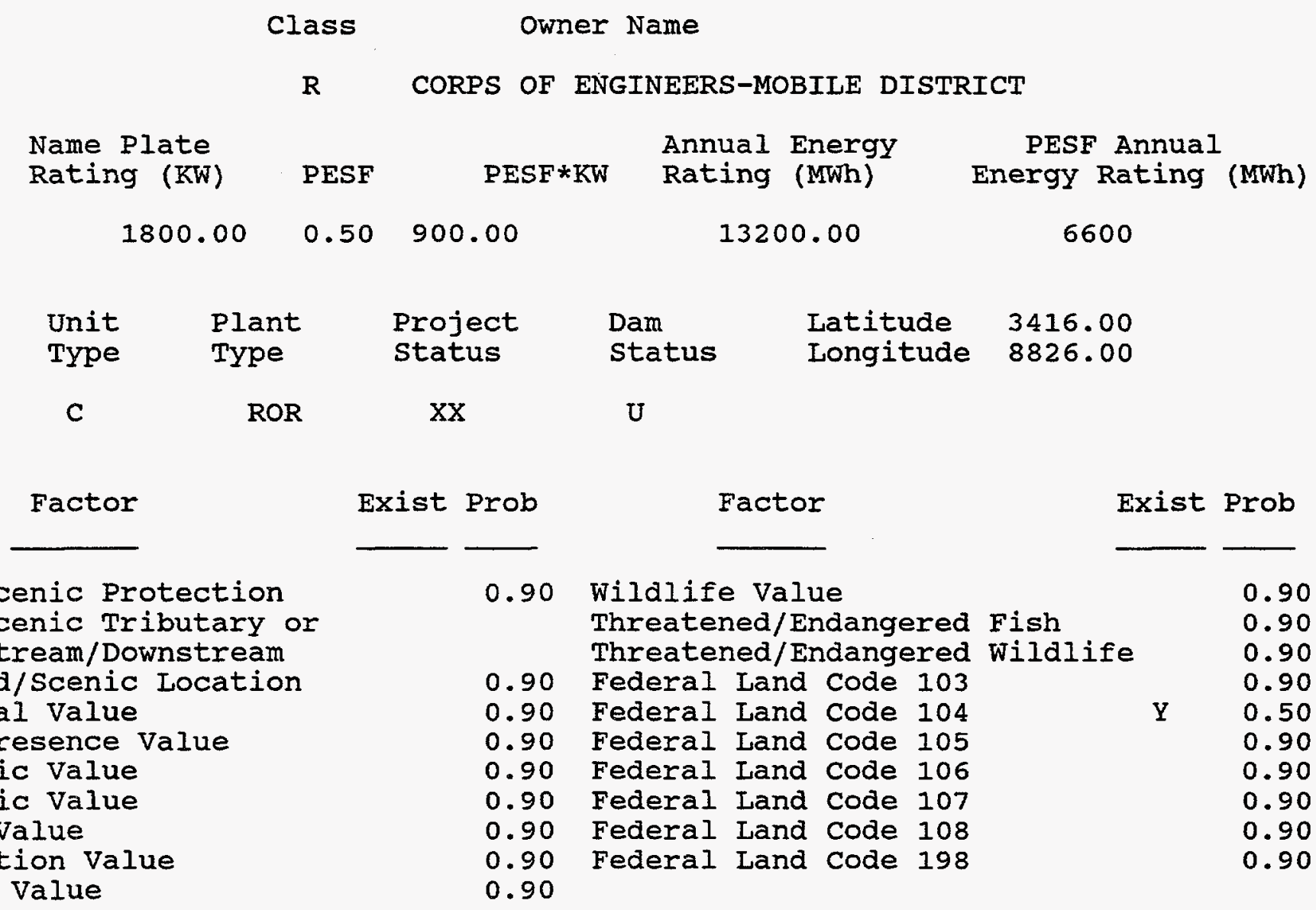

Wild/Scenic Protection

wild/Scenic Tributary or Upstream/Downstream wild/Scenic Location Cultural value

Fish Presence Value

Geologic Value

Historic Value

other Value

Recreation Value

Scenic Value

0.90

0.90

0.90

0.90

0.90

0.90

0.90

0.90 
DATE: $10 / 17 / 97$

FERC

Number

09210 TENN-TOM LOCK E

County Name

ITAWAMBA

Class

Owner Name

R CORPS OF ENGINEERS-MOBILE DISTRICT

River Basin

TOMBIGBEE RIVER BASIN

PAGE NO: 7
State

Name

TOMBIGBEE $\mathrm{R}$

MS

stream

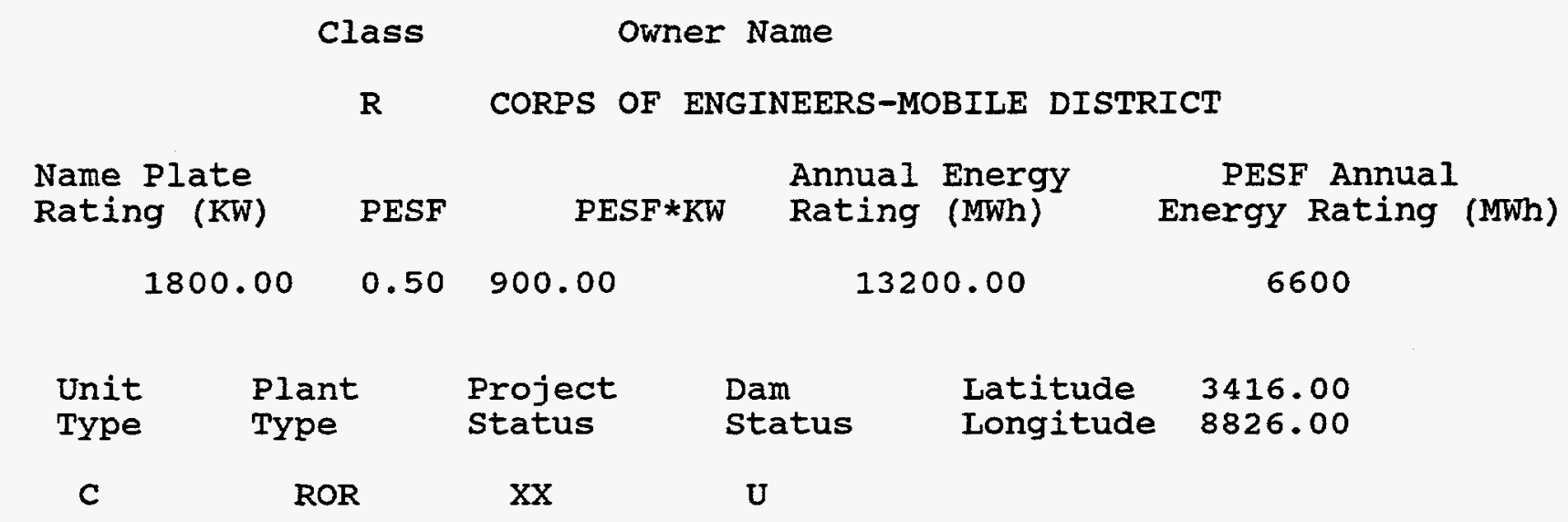

Factor

Exist Prob

Factor

Exist Prob

Wild/Scenic Protection

wild/Scenic Tributary or

0.90 Wildife Value

0.90

Upstream/Downstream

Threatened/Endangered Fish

0.90

wild/Scenic Location

Threatened/Endangered Wildlife

0.90

0.90 Federal Land Code 103

0.90 Federal Land Code 104

0.90

Cultural value

0.90 Federal Land Code 105

0.50

Fish Presence Value

0.90 Federal Land Code 106

0.90

Geologic Value

0.90 Federal Land Code 107

0.90

Historic Value

0.90 Federal Land Code 108

0.90

Other Value

0.90

Federal Land Code 198

0.90

0.90

Scenic Value

0.90 

R E S O U R C E
D A T A B A S E
L I S T I N G

DATE: $10 / 17 / 97$

PAGE NO: 8

FERC

Number

Plant Name

stream

State

09211 TENN-TOM LOCK C

TOMBIGBEE $\mathrm{R}$

Name

MS

county Name

ITAWAMBA
River Basin

TOMBIGBEE RIVER BASIN

\section{Class Owner Name}

R CORPS OF ENGINEERS-MOBILE DISTRICT

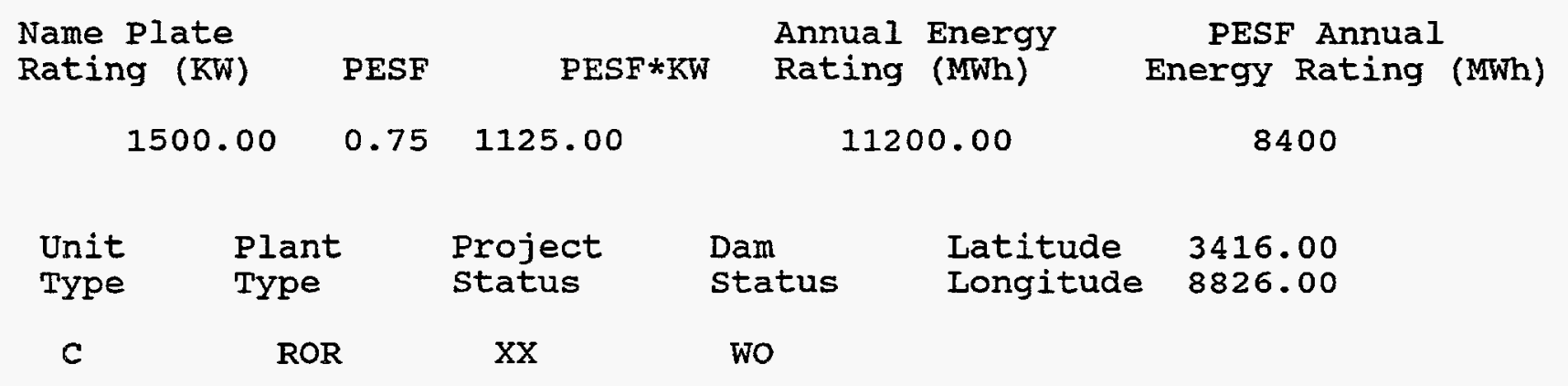

Factor

Exist Prob

Factor

Exist Prob

Wild/Scenic Protection

Wild/Scenic Tributary or

0.90 Wildlife Value

Threatened/Endangered Fish

Upstream/Downstream Threatened/Endangered Wildlife

Wild/Scenic Location

Cultural value

Fish Presence Value

Geologic Value

Historic Value

other value

Recreation value

Scenic Value

0.90 Federal Land Code 103

0.90 Federal Land Code 104

0.90 Federal Land Code 105

0.90 Federal Land Code 106

0.90 Federal Land Code 107

0.90 Federal Land Code 108

0.90 Federal Land Code 198

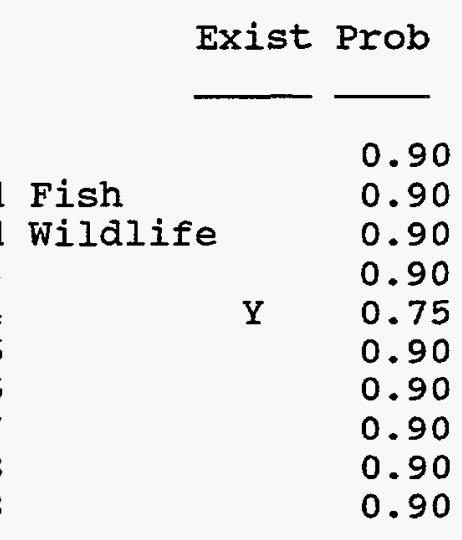


DATE: $10 / 17 / 97$

FERC

Number

09212 TENN-TOM LOCK B(2)

County Name

ITAWAMBA
PAGE NO: 9

state

Name

TOMBIGBEE $R$

MS

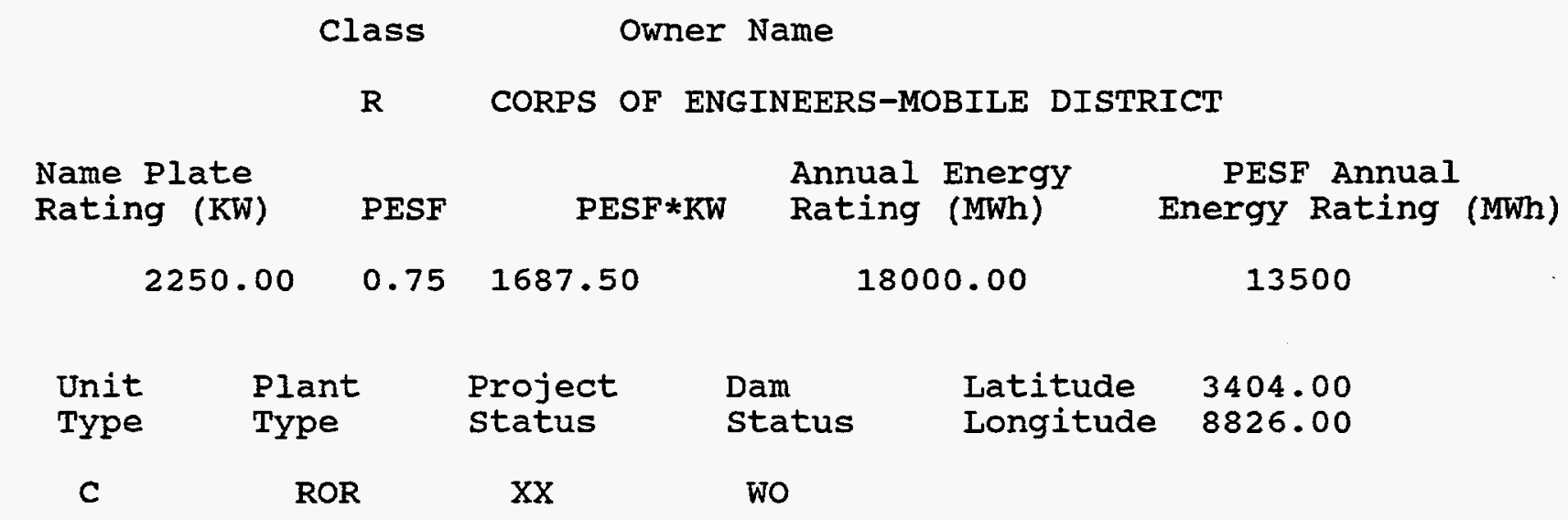

Factor

Wild/Scenic Protection

wild/scenic Tributary or Upstream / Downstream wild/Scenic Location Cultural value Fish Presence Value Geologic value

Historic Value other Value

Recreation value

Scenic Value
Exist Prob

0.90

Wildlife Value Threatened/Endangered Fish Threatened/Endangered Wildlife

Exist Prob

Factor

$\begin{array}{lll}0.90 & \text { Federal Land Code } 103 \\ 0.90 & \text { Federal Land Code } 104\end{array}$

0.90 Federal Land Code 105

0.90 Federal Land Code 106

0.90 Federal Land Code 107

0.90 Federal Land code 108

0.90 Federal Land Code 198 
DATE: $10 / 17 / 97$

\section{FERC}

Number

09213
Plant Name

TENN-TOM LOCK A

County Name

MONROE
PAGE NO: 10

State

Name

TOMBIGBEE R

MS

$$
\begin{array}{cc}
\text { Class } & \text { Owner Name } \\
\text { R } & \text { CORPS OF ENGINEERS-MOBILE DISTRICT }
\end{array}
$$

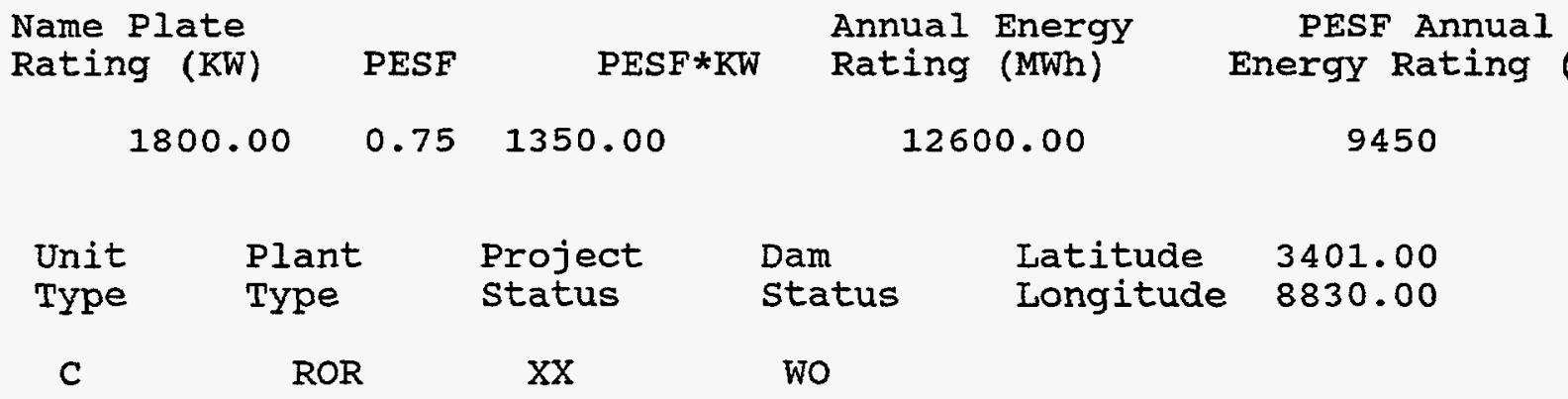

Factor

Exist Prob

Factor

Exist Prob

0.90 Wildlife Value Threatened/Endangered Fish Threatened/Endangered Wildife

0.90

Wild/Scenic Protection wild/Scenic Tributary or Upstream/Downstream wild/Scenic Location Cultural Value Fish Presence Value Geologic Value Historic Value other value Recreation value Scenic value $\begin{array}{ll}0.90 & \text { Federal Land Code } 103 \\ 0.90 & \text { Federal Land Code } 104\end{array}$

0.90 Federal Land Code 105

0.90 Federal Land Code 106

0.90 Federal Land Code 107

0.90 Federal Land Code 108

0.90 Federal Land Code 198
0.90

0.90

0.90

Y

0.75

0.90

0.90

0.90

0.90

0.90 

R E S O U R C E
D A T A B A S E
L I S T I N G

DATE: $10 / 17 / 97$

PAGE NO: 11

FERC

Number

Plant Name

stream

State

Name

10410 ARKABUTLA

COIDWATER R

MS

County Name

DE SOTO
River Basin

MINOR RIVER BASINS

Class Owner Name

R CORPS OF ENGINEERS-VICKSBURG DISTR

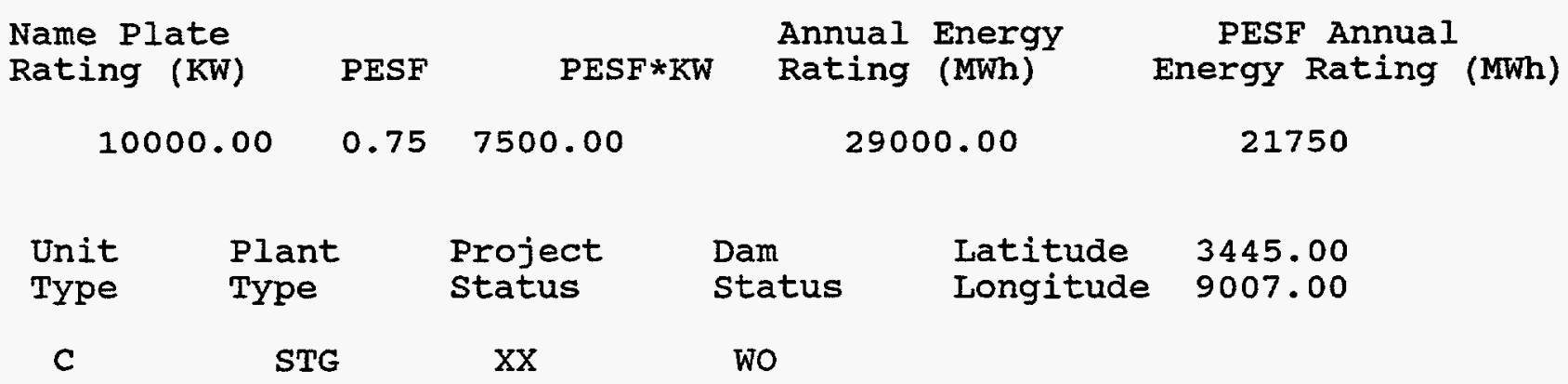

Factor

Wild/Scenic Protection

wild/Scenic Tributary or Upstream/Downstream wild/Scenic Location Cultural Value

Fish Presence Value

Geologic Value

Historic Value

other value

Recreation Value

Scenic Value
Exist Prob

Factor

Exist Prob

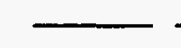

$$
0.90
$$

Wildife Value Threatened/Endangered Fish Threatened/Endangered Wildlife

0.90 Federal Land Code 103

0.90 Federal Land Code 104

0.90 Federal Land Code 105

0.90 Federal Land Code 106

0.90 Federal Land Code 107

0.90 Federal Land Code 108

0.90 Federal Land Code 198

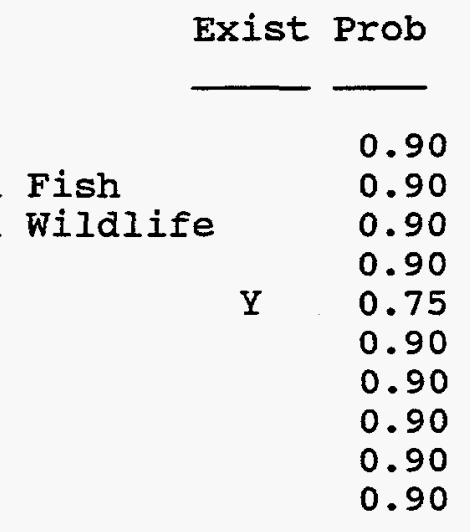


FERC

Number

10411 ENID
YACONA R

River Basin

YALOBUSHA

Class Owner Name

MINOR RIVER BASINS state Name

MS

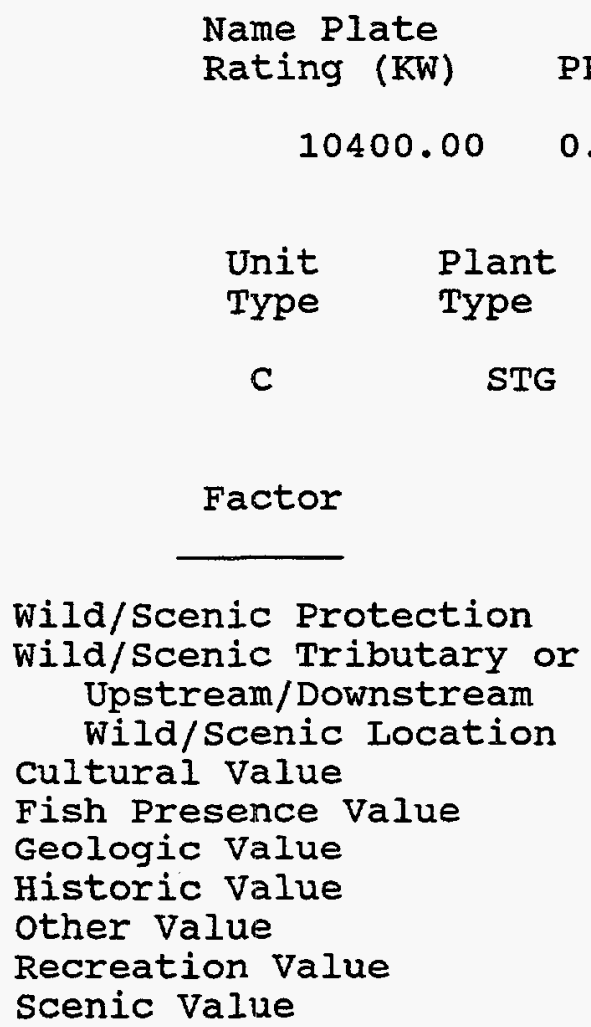

STG

Unit
Type

C

Factor

wild/Scenic Tributary or Upstream/Downstream wild/Scenic Location Cultural value

Fish Presence Value Geologic Value Historic Value other value Recreation Value Scenic Value

Exist Prob
0.90

Wildlife Value Threatened/Endangered Fish

$\begin{array}{clll}\text { Project } & \text { Dam } & \text { Latitude } & 3409.00 \\ \text { Status } & \text { Status } & \text { Longitude } & 8954.00 \\ \text { XX } & \text { wo } & & \end{array}$

Annual Energy Rating (MWh)

PESF Annual Energy Rating (MWh)

18750

Factor

Exist Prob Threatened/Endangered Wildlife

0.90 Federal Land Code 103

0.90 Federal Land Code 104

0.90 Federal Land Code 105

0.90 Federal Land Code 106

0.90 Federal Land Code 107

0.90 Federal Land Code 108

0.90 Federal Land Code 198

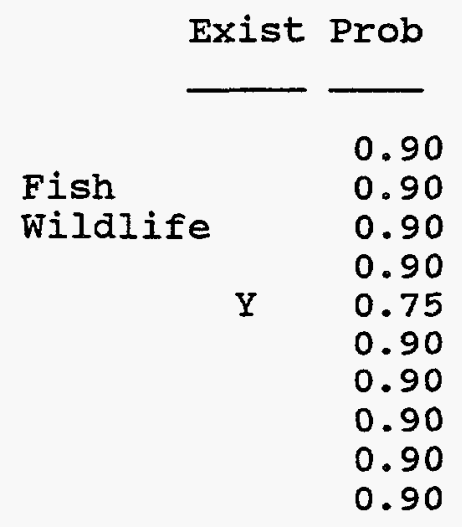



R E S O U R C E
D A T A B A S E
I I S T I N G

DATE: $10 / 17 / 97$

PAGE NO: 13

FERC

Number

Plant Name

stream

State

10412 GRENADA

YALOBUSHA $R$

Name

MS

County Name

River Basin

GRENADA

MINOR RIVER BASINS

Class Owner Name

$R$ CORPS OF ENGINEERS-VICKSBURG DISTR

Name Plate

Annual Energy

Rating (KW)

PESF

PESF*KW

Rating (MWh)

PESF Annual

17000.00

$0.75 \quad 12750.00$

50000.00

Energy Rating (MWh)

Unit Plant

Type Type

Project

status

Dam

status

Latitude

Longitude

$\mathrm{XX}$

WO

Factor

Wild/Scenic Protection

wild/Scenic Tributary or Upstream/Downstream wild/Scenic Location Cultural value Fish Presence Value Geologic Value Historic Value other Value

Recreation value Scenic value
Exist Prob

0.90

Wildlife value Threatened/Endangered Fish Threatened/Endangered Wildlife

0.90 Federal Land Code 103

0.90 Federal Land Code 104

0.90 Federal Land Code 105

0.90 Federal Land code 106

0.90 Federal Land Code 107

0.90 Federal Land Code 108

0.90 Federal Land Code 198
3349.00

37500

8946.00 
FERC

Number

10413
Plant Name

SARDIS

County Name

PANOLA stream

LITTLE TALLAHATCHIE $R$

River Basin

MINOR RIVER BASINS
State

Name

MS

\section{Class Owner Name}

R CORPS OF ENGINEERS-VICKSBURG DISTR

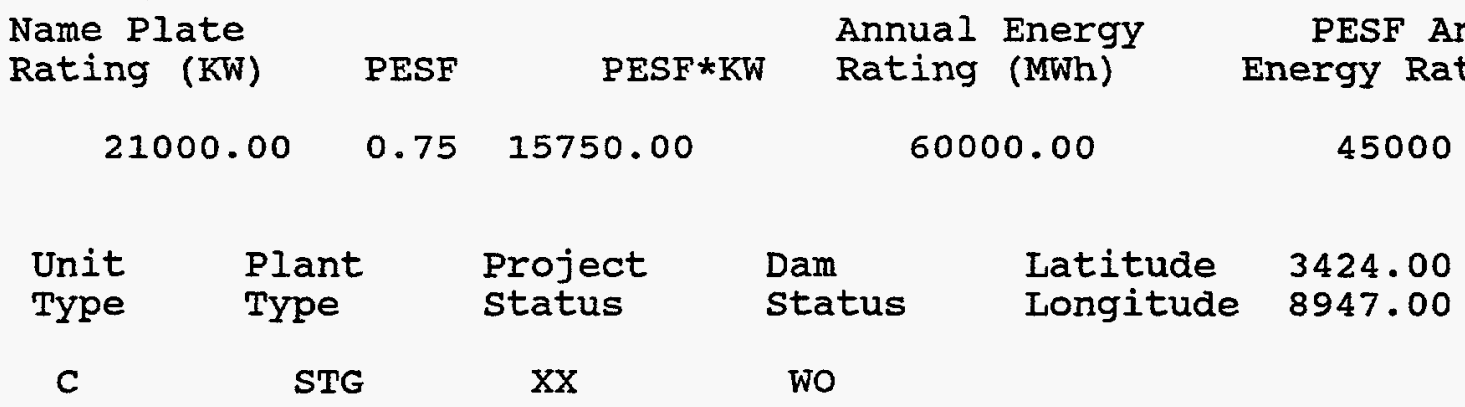

Factor

Exist Prob

\subsection{0}

Wild/Scenic Protection

wild/Scenic Tributary or Upstream/Downstream wild/Scenic Location

Cultural value

Fish Presence Value

Geologic value

Historic Value

other Value

Recreation Value

Scenic Value
Factor

Exist Prob

0.90
0.90
0.90
0.90
0.75
0.90
0.90
0.90
0.90
0.90


DATE: $10 / 17 / 97$

FERC

Number

Plant Name

MSO01 ARCHUSA CREEK

County Name

CLARKE

Class

Owner Name

M PAT HARRISON WTRWY DISTRICT

Name Plate

Rating (KW)

PESF

$\mathrm{PESF} * \mathrm{KW}$

Annual Energy

Rating (MWh)

320.00

110.00

0.50

55.00

Project Status

Unit
Type

Plant

Type

ROR

Factor

Wild/Scenic Protection

wild/Scenic Tributary or Upstream/Downstream wild/Scenic Location Cultural value

Fish Presence Value

Geologic Value

Historic Value

other Value

Recreation value

Scenic Value

wo
Exist Prob

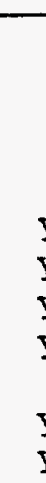

PAGE NO: 15

state

Name

MS
PESF Annual Energy Rating (MWh)

160

$\begin{array}{lll}\text { Dam } & \text { Latitude } & 0.00 \\ \text { Status } & \text { Longitude } & 0.00\end{array}$

Factor

0.90 Wildlife Value Threatened/Endangered Fish Threatened/Endangered Wildlife

0.90 Federal Land Code 103

0.75 Federal Land Code 104

0.75 Federal Land Code 105

0.90 Federal Land code 106

0.75 Federal Land Code 107

0.90 Federal Land Code 108

$\mathrm{Y} \quad 0.75$ Federal Land Code 198
Exist Prob

Y
0.75

0.90

0.90

0.90

0.90

0.90

0.90

0.90

0.90

0.90 
DATE: $10 / 17 / 97$

FERC

Number

Plant Name

MSOO2 FLINT CREEK DAM

County Name

STONE

Class

Owner Name

M

PAT HARRISON WTRWY DISTRICT

Name Plate

Rating (KW)

60.00

PESF

$\mathrm{PESF} * \mathrm{KW}$

Annual Energy

Rating (MWh)

173.00

$0.50 \quad 30.00$

Project

Status

Type

Plant

Type

ROR

Factor

Exist Prob

0.90

Wildlife Value

Threatened/Endangered Fish

Threatened/Endangered Wildlife

Latitude $\quad 3051.00$

Status

Longitude

8849.00
State

Name

MS
PESF Annual Energy Rating (MWh)

86.50
Wild/Scenic Protection

wild/Scenic Tributary or

Upstream/Downstream wild/Scenic Location Cultural value

Fish Presence Value

Geologic Value

Historic Value

other value

Recreation Value

Scenic Value $\begin{array}{ll}0.90 & \text { Federal Land Code } 103 \\ 0.90 & \text { Federal Land Code } 104\end{array}$

$\begin{array}{lll}\mathrm{Y} & 0.75 & \text { Federal Land Code } 105 \\ \mathrm{Y} & 0.90 & \text { Federal Land Code } 106\end{array}$

0.90 Federal Land code 107

0.90 Federal Land Code 108

$\mathrm{Y} \quad 0.75$ Federal Land Code 198

$\begin{array}{ll}\mathrm{Y} & 0.75 \\ \mathrm{Y} & 0.90\end{array}$
Exist Prob

$Y$

0.75

0.90

0.90

0.90

$\mathrm{Y}$

0.75

0.90

0.90

0.90

0.90

0.90 
DATE: $10 / 17 / 97$

FERC

Number

Plant Name

MSO03 HARLESTON

county Name

GEORGE

Class

Owner Name
PAGE NO: 17

State

Name

MS
ESCATAWPA R

River Basin

PASCAGOULA RIVER BASIN

\begin{tabular}{|c|c|c|c|c|c|}
\hline \multicolumn{2}{|c|}{ Class } & \multicolumn{2}{|c|}{ Owner Name } & & \multirow[b]{2}{*}{$\begin{array}{l}\text { PESF Annual } \\
\text { Energy Rating (MWh) }\end{array}$} \\
\hline $\begin{array}{l}\text { Name Plate } \\
\text { Rating (KW) }\end{array}$ & PESF & $\mathrm{PESF} * \mathrm{KW}$ & $\begin{array}{l}\text { Annual } \\
\text { Rating }\end{array}$ & $\begin{array}{l}\text { Energy } \\
(\text { MWh })\end{array}$ & \\
\hline 11000.00 & 0.90 & 9900.00 & \multicolumn{2}{|c|}{20200.00} & 18180 \\
\hline $\begin{array}{l}\text { Unit } \\
\text { Type }\end{array}$ & & $\begin{array}{l}\text { Project } \\
\text { status }\end{array}$ & $\begin{array}{l}\text { Dam } \\
\text { Status }\end{array}$ & $\begin{array}{l}\text { Latitude } \\
\text { Longitude }\end{array}$ & $\begin{array}{l}3044.00 \\
8828.00\end{array}$ \\
\hline$c$ & & & $\mathrm{U}$ & & \\
\hline
\end{tabular}

Factor

Wild/Scenic Protection

Wild/Scenic Tributary or Upstream/Downstream wild/Scenic Location

Cultural Value

Fish Presence Value

Geologic Value

Historic Value

other value

Recreation Value

Scenic Value
Exist Prob

Factor

Exist Prob

0.90

Wildlife value Threatened/Endangered Fish Threatened/Endangered Wildlife

0.90 Federal Land Code 103

0.90 Federal Land Code 104

0.90 Federal Land code 105

0.90 Federal Land code 106

0.90 Federal Land Code 107

0.90 Federal Land Code 108

0.90 Federal Land Code 198
0.90

0.90

0.90

0.90

0.90

0.90

0.90

0.90

0.90

0.90 
DATE: $10 / 17 / 97$

PAGE NO: 18

FERC

Number

Plant Name

stream

State

MSOO4 YOCKANOOKANY

YOCKANOOKANY R, PEARI R

Name

County Name

IEAKE

River Basin

PEARL RIVER BASIN

class Owner Name

\begin{tabular}{|c|c|c|c|c|c|c|}
\hline \multicolumn{2}{|c|}{$\begin{array}{l}\text { Name Plate } \\
\text { Rating (KW) }\end{array}$} & PESF & PESF *KW & \multicolumn{2}{|c|}{$\begin{array}{l}\text { Annual Energy } \\
\text { Rating (MWh) }\end{array}$} & $\begin{array}{l}\text { PESF Annual } \\
\text { Energy Rating (MWh) }\end{array}$ \\
\hline \multicolumn{2}{|c|}{3300.00} & 0.90 & 2970.00 & \multicolumn{2}{|c|}{10100.00} & 9090 \\
\hline $\begin{array}{l}\text { Unit } \\
\text { Type }\end{array}$ & $\begin{array}{l}\text { P1 } \\
\text { TY }\end{array}$ & & $\begin{array}{l}\text { Project } \\
\text { status }\end{array}$ & $\begin{array}{l}\text { Dam } \\
\text { Status }\end{array}$ & $\begin{array}{l}\text { Latitude } \\
\text { Longitude }\end{array}$ & $\begin{array}{l}0.00 \\
0.00\end{array}$ \\
\hline C & & & & $\mathrm{U}$ & & \\
\hline
\end{tabular}

Factor

Exist Prob

Factor

Exist Prob

Wild/scenic Protection

Wild/Scenic Tributary or

0.90 Wildife Value

Threatened/Endangered Fish

MS

Upstream/Downstream

wild/Scenic Location

Threatened/Endangered Wildlife

0.90

0.90

0.90 Federal Land Code 103

0.90

0.90 Federal Land code 104

0.90

0.90 Federal Land Code 105

0.90

Fish Presence Value

0.90 Federal Land Code 106

0.90

0.90 Federal Iand Code 107

0.90

Historic Value

0.90 Federal Land Code 108

0.90

other value

Recreation Value

0.90

Federal Land Code 198

0.90

0.90

0.90 
DATE: $10 / 17 / 97$

FERC

Number

MS005

YOUNGTON

County Name

WARREN

Plant Name

County Name

WARREN

class
PAGE NO: 19

State

Name

BIG BLACK $R$

MS

River Basin

MINOR RIVER BASINS

Owner Name

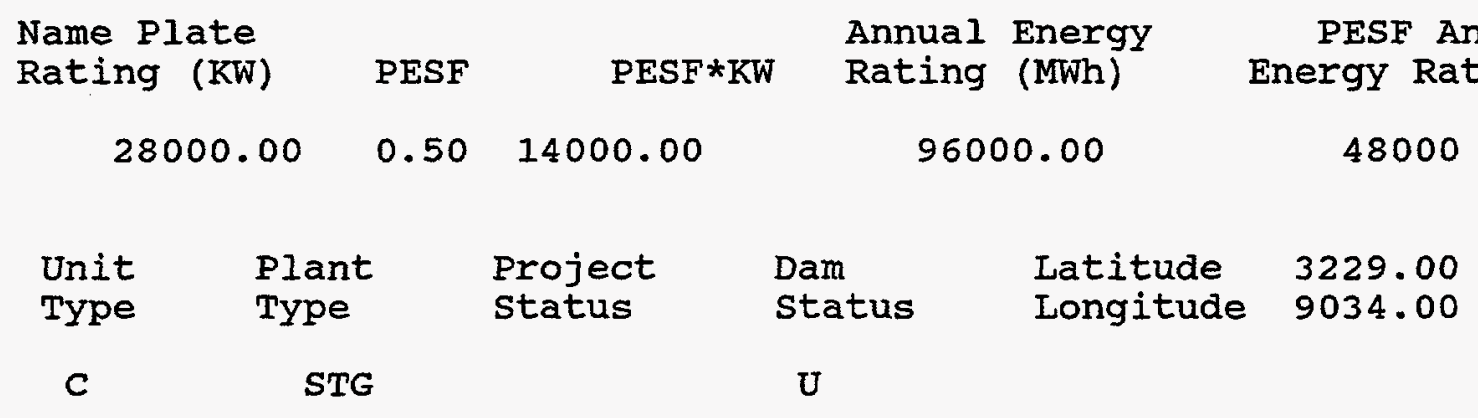

Factor

Exist Prob

Factor

Exist Prob

Wild/Scenic Protection

wild/Scenic Tributary or

Upstream/Downstream

wild/Scenic Location

Cultural value

Fish Presence Value

Geologic Value

Historic Value

Other Value

Recreation Value

Scenic Value
Exist Prob

0.90 Wildlife Value

Threatened/Endangered Fish

Threatened/Endangered Wildlife

0.90 Federal Land code 103

$Y \quad 0.75$ Federal Land Code 104

$Y \quad 0.75$ Federal Land code 105

0.90 Federal Land Code 106

$Y \quad 0.75$ Federal Land code 107

0.90 Federal Land Code 108

$\mathrm{Y} \quad 0.75$ Federal Land Code 198
$\mathrm{Y} \quad 0.90$ 